\section{A NOTE ON THE EARLY TREATMENT OF PROTRUDING INCISOR.}

BY J. F. COLYER, M.R.C.S., L.D.S.R.O.S. ENG., DENTAL SURGEON AND LECTURER ON DENTAL SURGERY TO OHARING CROSS HOSPITAL; SURGEON AND DEAN TO THE BOYAL DENTAL HOSPLTAL, LONDON.

Protrusion of the upper incisor teeth is a deformity only too often seen amongst the children of the present day, and it is one on which dental practitioners are by no means in agreement as to the best method of treatment. In a few cases the protrusion of the teeth is the direct result of the use of the baby comforter, of lip sucking, or a somewhat similar habit ; in the majority of instances, however, the protrusion is accompanied by an abnormal growth of the jaws and dental arches. It is to this latter class I propose to refer.

Now, although there is a certain amount of obscurity about the etiology and pathology of these cases, there are, however, certain facts generally accepted, and to these brief allusion will be made, as they have an important bearing on the question of treatment. In the first place, attention must be drawn towards a well-recognised factnamely, that the teeth themselves are formed during the first three years of life, and are arranged, one might say, in a crowded manner in the substance of the bone. If the teeth could be taken and fixed in the mouth the arch of the jaws would be found quite inadequate for the correct arrangement of the teeth. If orderly development of the jaws takes place then by the time the teeth erupt there is room for their proper and correct arrangement. This orderly development is under modern conditions interfered with, mainly by lack of function of the jaws, with the result that there is an interference with the growth of the body of the jaw. What is the result? A stru ggle takes place on the part of the teeth for room in the jaw, and either the incisors and canines together, at times the premolars, are crowded into irregular positions, or the anterior teeth are pushed forwards, causing superior protrasion. Why in the one case simple crowding results and in the other superior protrusion is not clear.

This lack of development would seem to affect the maxilla and mandible unequally. Clinical evidence shows that the maxilla suffers more frequently and more severely than the mandible, and for this reason the growth of the mandible is influenced by the function of mastication, whereas that of the maxilla is in addition considerably influenced by the proper performance of the nasal function-in other words, in nasal obstruction the antrum and premaxilla do not develop properly, and this interferes in a marked manner with the proper growth of the maxillæ. In the majority of cases of superior protrusion I consider the major lack of development is in the maxilla and not the mandible. This is, of course, the reverse of the position taken by those who lay so much stress upon the question of occlusion. In a few, but a very few, cases the protrusion is apparent not real-that is to say, the maxillæ are normal, but the mandible is deficient in development.

Another point to be considered is the rapid increase in the protrusion of the teeth as the child approaches the age of 12. This is due to the action of the canines in erupting. The four incisors may be pushed forward, the teeth maintaining a good curve, or the laterals may be forced under cover of the centrals, giving rise to what is sometimes styled "a rabbit-shaped" mouth.

To sum up, the points to be emphasised are: 1 . Protrusion of the superior teeth is usually accompanied by some lack of development of the bone, producing a want of relationship between the teeth and the jaw. 2. The maxilla is more often affected than the mandible. 3. The protrusion gets rapidly worse with the eruption of the canines.

What line of treatment do these facts indicate? Clearly, extraction of teeth to relieve the crowding and still further extraction at an early age. This method is at variance with the views of many practitioners who urge that no attempt at correction should be made until after the eruption of the second permanent molars and canines.

Let us consider for a few moments the disadvantages of delaying treatment; the advantages it is difficult to see. 1. The canine in erupting will move forward, increasing the protrusion of the incisors and also the crowding of their roots. 2. When treatment is commenced the canine must be retracted by mechanical means, and in this process instead of moving bodily back and so giving true relief to the pressure in the front of the month it will only swing backwards, leaving the upper end of the root in much the same position as it erupted. The result of this will be a constant tendency for the protrusion when corrected to relapse, simply because the roots of the anterior teeth will remain more or less crowded. 3 . The retraction of the incisors will occupy a greater length of time, partly because there will be more protrusion to overcome and partly because the alveolar process will be more resistant. 4. Owing to the prolonged mechanical treatment disorganisation of molar and premolar occlusion often ensues.

If treatment is carried out before the eruption of the canines these teeth will erupt well back, pressure will immediately be taken off the incisors, the protrusion will not increase, and the amount of mechanical treatment will be only slight. Still further, the teeth come into good align. ment and the tendency to relapse is reduced to the minimum. If early treatment is adopted the room for the canines should be made directly the lateral incisors are in place by removing the nnerupted first premolars. For this operation an anæsthesia of longer duration than ordinary nitrous oxide is necessary. After a little practice ethyl chloride will usually be found sufficient but if the case is likely to be difficult then ether should be used.

The most suitable instrument for removing the teeth is a pair of upper root forceps with long slender blades. The deciduous molar is first removed. In attempting the removal of the premolar the inner blade of the forceps must be kept well inwards, the blades being thrust well upwards, and extractive force made in an inward direction. The occluding surface of the erupting premolar is directed slightly inwards and unless the precaution suggested is taken the inner blade of the forceps impinges on the occluding surface and does not grip the inner aspect.

With regard to the treatment of the wound, the best course seems to be to keep the mouth clean by means of an antiseptic wash. If much swelling ensues the use of hot fomentations will generally bring relief.

FIG. 1.

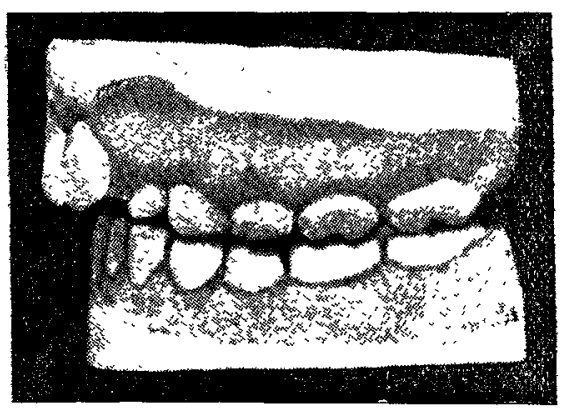

Case 1. Before treatment.

FIG. 2.

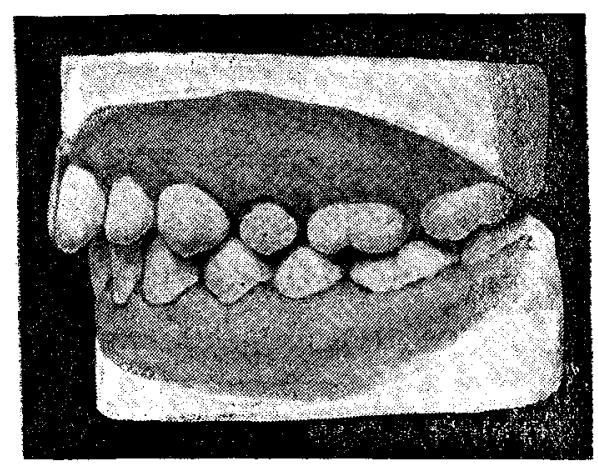

Case 1. After treatment.

After the removal of the premolars the case is left alone nntil the canines have fully erupted, the patient being made to keep the lower lip in front of the upper teeth. Occasionally this simple expedient is sufficient to bring about a complete cure of the case. The effect of the removal of the pressure of the canines on the incisors is interesting. The laterals soon show a tendency to travel backward, pointing to a complete relief of the crowding of the anterior teeth. The incisors are eventually brought in 
with a simple mechanical contrivance, the teeth being/for the first molars and the canines to be correctly related, retained in position until after the second permanent molars have erupted.

IWith regard to the treatment of the lower teeth, one is guided by the condition existing. If crowding is present then the : first premolars are removed, but the time for

FIG. 3.

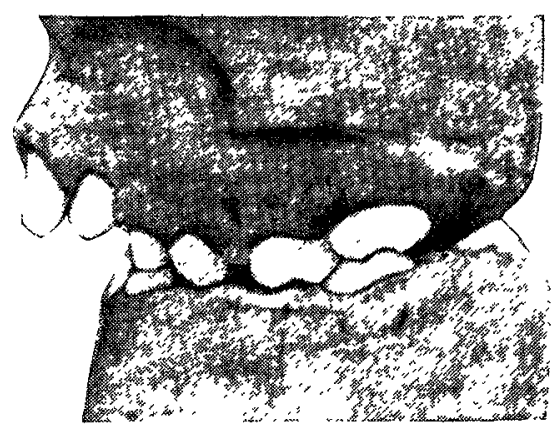

Case 2. Before treatment.

FIG. 4.

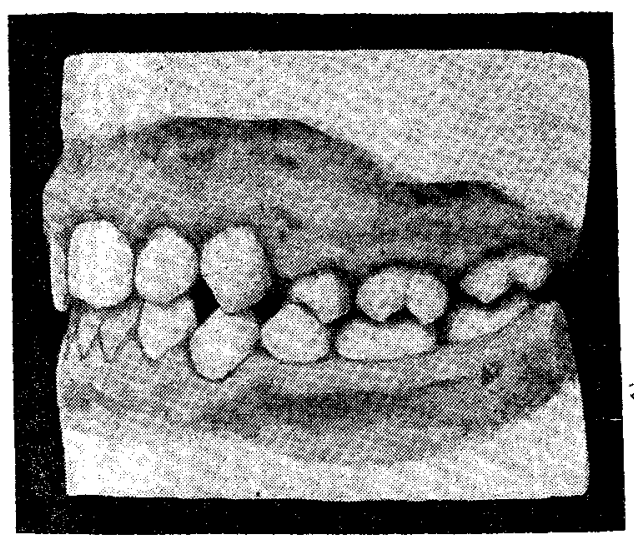

Case 2. After treatment.

removing them is left until the commencement of eruption of the canines. But little is gained by removing the mandibular premolars at quite an early age, as is the case in the maxilla.

Cases of superior protrusion treated in the way suggested do extremely well; there is no tendercy to recur, the amount of mechanical treatment is very little, and the occlusion of the premolars and molars is good. In the figures are shown the models of two cases before, and the same cases after, treatment.

Much has been said about the tendency of this type of irregularity when treated to relapse. There will be little if an tendency to relapse if we can: (1) insure that the lower lip in occlusion passes in front of the upper incisors; (2) insure that the lower incisors do not press unduly upon the backs of the upper incisors; and (3) prevent crowding of the upper incisors and canines. The less we are unable to overcome these difficulties the greater will be the tendency to relapse.

\section{THE TREATMENT OF MALOCCLUSION (ORTHODONTIA).}

\section{By H. C. HIGHTON, L.D.S. R.C.S. ENG., D.D.S. PenN.}

THE fundamental principle of orthodontia is that we have a thorough understanding of normal occlusion and all that the term implies. It is necessary to appreciate the normal relationship of the teeth of the mandible with those of the maxilla, how each individual tooth is dependent not only apon its fellows mesially and distally but upon the opposing teeth and all the teeth collectively to maintain harmony, and then only is it possible to understand thoroughly any of the various types or deviations from the normal. From a study of skulls of past and present races normally the mesio buccal cusp of the upper first molar occludes between and buccally to the mesio- and disto-buccal cusps of the lower first molar. The mesial incline of the upper canine occludes with the distal incline of the lower canine, the other teeth if regular being then normally related; it is quite necessary otherwise malocclusion and alteration of the facial lines mast result.

Classification.- The classification of malocolusion as suggested by Dr. E. H. Angle ${ }^{1}$ is based upon the variation from the harmonious relationship known as normal occlusion three great classes being represented, the first having normal mesio-distal relation of the arches, the second having the lower arch distal to normal in its relation to the upper either unilaterally or bilaterally, and the third having the lower arch mesial to normal in relation to the upper either unilaterally or bilaterally. The great majority of cases of malocclusion belong to the first class, the second class comprising a small percentage, and the third a still smaller percentage of the various existing cases of malocclusion. In the first class the teeth may be in any possible position of malocclusion which would be consistent with a normal antero-posterior relationship between the arches of the teeth. For example, any of the incisors and canines may be in labial or lingual occlusion, the bicuspids or molars in buccal or lingual occlusion. There may be protrusion of the anterior part of the upper arch or retrusion of the anterior part of the lower arch without disturbing the normal anteroposterior relationship.

The treatment in all cases of malocclusion is therefore simply the placing in harmony of the inclined planes of the teeth and establishing of normal occlusion. The principle underlying this treatment, which is advocated in "orthodontia," is expansion of the arch as opposed to extraction and the consequent narrowing of the arch and alteration of the facial lines. Many people come under our observation with mutilated mouths and abnormal facial expressions that have been brought about by extraction of the teeth, often to prevent some developing form of malocclusion. The ideal to be kept in view is the attainment of normal occlusion which presupposes the full complement of teeth.

There are, briefly, at least three important reasons for the expansion of the dental arch. First, to permit malposed teeth to assume their correct alignment; second, to improve the facial lines; third, to endeavour to broaden the nasal chambers and so increase the capacity for nasal breathing. The latter is naturally a very important consideration, as it concerns the future health and welfare of the patient. Expansion of the dental arch to be most effective should be done as early in life as possible; gradual force exerted while the patient is young encourages the natural development of these parts. In treatment it is also necessary to rotate the teeth, move them labially or lingually, mesially or distally, any of which movements are most easily accomplished by means of the clamp band, expansion arch, and wire ligatures. As the ultimate success of the treatment naturally depends upon the ease of retention of the teeth in the corrected position-viz., in normal occlusion-it will readily be understood how essential it is to retain the full number of the teeth, since only by that means can the inclined planes of the teeth be placed in correct normal relationship which if accomplished will simplify to a very great extent the method and length of time of the retention. Extraction, therefore, should always be avoided if possible, as not only does it destroy the possibility of obtaining an ideal result but also renders more difficult the final retention.

We find that in the majority of the cases of malocclusion the arch is narrow, the teeth crowded together, overlapping, and malposed to a greater or less degree, and the forces which acted in bringing about this malformation are equally powerful in maintaining and exaggerating this condition when once it has been established. Orthodontia aims at placing every tooth in its normal position in relation with its fellows and those of the opposite arch and retaining them in that position until the muscles, alveolus, and tissues of the neighbouring region have accommodated themselves to the altered conditions. The means usually employed to bring about this result is a fixed appliance which is arranged as follows: clamp bands are secured to the first upper and lower molar teeth; these consist of a band with a horizontal tube on the buccal side and a nut and screw on the lingual side to tighten the band to the required size. The ends of an elastic wire arch, the expansion arch, are inserted into the buccal tubes and the arch is ligated to the teeth by means of fine wire ligatures, the expansion being brought about by the arch having an outward spring, while the ligatures can be oecasionally tightened. The teeth may

Treatment of Malocclusion of the Teeth, 1907. 